\title{
Spatial Learning Paradigm Can Increase Post-Stress Total Time of REM Sleep in Immobilized Rats
}

\author{
Fatemeh Erfani Sharifian $^{1}$ (D), Farideh Bahrami2* ${ }^{*}$, Zahra Bahari2 ${ }^{\text {iD }}$
}

1. Neuroscience Research Center, Baqiyatallah University of Medical Sciences, Tehran, Iran

2. Dept. of Physiology and Medical Physics, Faculty of Medicine, Baqiyatallah University of Medical Sciences, Tehran, Iran

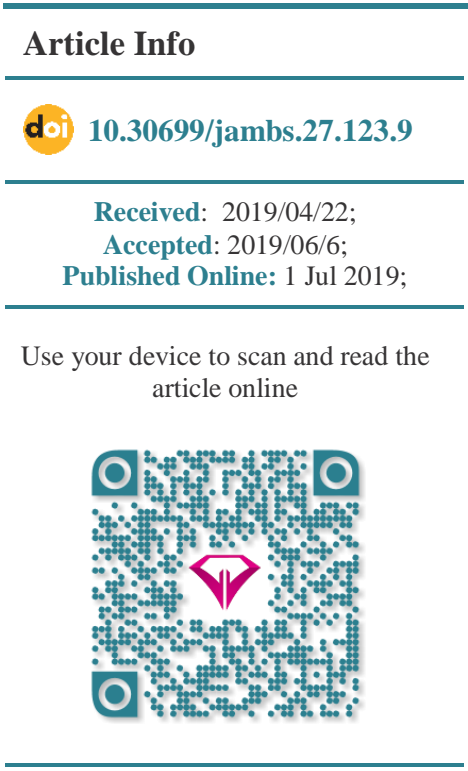

Corresponding Information: Farideh Bahrami, Dept. of Physiology and Medical Physics, Faculty of Medicine, Baqiyatallah University of Medical Sciences, Tehran, Iran

E-Mail: f.bahrami@bmsu.ac.ir

\begin{abstract}
Background \& Objective: Stress contributes to sleep-wake behavior in all animals. It seems that factors such as learning and memory processes can improve sleep disorders. Therefore, the present study was conducted to determine the effects of stress and learning (spatial memory) on total post-stress REM, NREM and waking time in rats.
\end{abstract}

Materials \& Methods: Adult rats ( $\mathrm{n}=21$ ) were divided into 3 groups; group 1 received only immobilization stress; group 2 was subjected only to the learning process (Barnes maze); and group 3 underwent both the stress and learning conditions. For each rat, sleep signals were recorded for 2 hours for 3 consecutive days. After recording of sleep or awakening signals, animal subjected to immobilization stress for 2 hours in each day. Then, post-stress and post-learning signals were recorded for another 2 hours.

Results: Immobilization stress resulted in significant decrease in total REM sleep time. However, total time of NREM increased following stress. Performing the learning task resulted in a significant increase in post-learning REM time $(\mathrm{p}<0.05)$. Moreover, total NREM time did not change markedly following the learning process. Interestingly, the learning process significantly $(\mathrm{p}<0.05)$ decreased total time of awakening when compared with pre-learning condition. However, learning process could increase REM sleep significantly $(\mathrm{p}<0.05)$ after the stress condition has been administered.

Conclusion: Our data suggested that immobilization stress could not prevent REM sleep after a learning process has been administered. However, the completion of a learning process increased post-stress REM time. It seems that learning helps to prevent the inhibitory effects of stress on REM sleep.

\section{Keywords: Sleep, REM, Learning and Memory, Immobilization, Rat}

\author{
Copyright $\odot$ 2019, This is an original open-access article distributed under the terms of the Creative Commons Attribution-noncommercial 4.0 International License which permits \\ copy and redistribution of the material just in noncommercial usages with proper citation.
}

\section{Introduction}

Stress is a physical and emotional response to external stimuli; which is challenges homeostasis and induces a multi-system response (1-4). It is believed that stress is an important factor in a variety of health problems, such as disturbances in sleep pattern $(\mathbf{5 , 6 )}$. Ample studies have reported marked changes in sleep patterns under various stress paradigms (7,8). Sanford and Yang revealed that uncontrollable stress significantly decreased sleep, whereas controllable stress significantly increases sleep (9). The finding that stress increases sleep has been reported in several studies $(10,11)$. However, there are conflicting studies in this area. In a study on humans, Levin et al. (2002) identified that emotional stress decreases slow-wave sleep in the first sleep cycle (12). Similarly, unpredictable or uncontrollable foot shock shortens rapid eye movement (REM) sleep in rodents (6). It has been suggested that REM sleep is affected the most by stress $(13,14)$. Ample study reported many animal models for inducing stress in rodents. It is suggested that the most commonly used model of stress is immobilization (13). Immobilization stress is referred to as psychological stress, because it does not cause any pain. However, in our study, we changed this model slightly, as the animals were placed in the narrow boxes, with some stones placed on the floor (physical stress). There are various stressors in the environment that disturb sleep patterns (15). However, successfully coping with stressors can reduce sleep disturbances and, thus, favorably influence healthy sleep patterns. Furthermore, it has been shown that environmental factors, such as training perform a task, can increase REM sleep after the learning procedure. Scientists have emphasized the role of memory consolidation in REM sleep. Machida and colleagues in 2013 have been shown that predictive auditory cues can change post-stress sleep, using electroencephalography (EEG) recording. They identified that animals that successfully learned escape, but not animals with failed to do so, showed significantly increased post-stress REM. They suggested that enhanced REM has a 
critical contribution in the consolidation of learning (14). Therefore, to provide additional insights into this topic, the purpose of this study is to investigate whether the stress condition can impair the REM sleep increment after learning procedure, or on the other hand learning can improve post stress- sleep disturbances. We examined the interaction of prior stress and learning on total REM sleep, NREM sleep and also waking time in rats.

\section{Materials and Methods}

\section{Animals}

Adult male Wistar rats $(n=21)$, weighing 200$250 \mathrm{gr}$, were used in the present study. The animals were kept under standard laboratory conditions (temperature: $25 \pm 2^{\circ} \mathrm{C}, 12 \mathrm{hr}$ light dark cycle). The animals were given free access to standard diet or water ad libitum. After a one-week accommodation period, the animals were randomly divided into 3 groups ( $\mathrm{n}=7$ per group). These Group 1 was the stress group; group 2 was the learning group; and group 3 was the stress + learning group. The method of the current study was approved by bioethics committee of animal house in Baqiyatallah University of Medical Science, which is in accordance with the $\mathrm{NIH}$ guide lines for care of animals (Ethical Research Code: IR.BMSU.REC.1396.393).

\section{Experimental design}

Experimental design is shown in Figure 1. As stated above, the animals divided into 3 groups $(n=7$ per group). Group 1 received only immobilization stress, group 2 was subjected to only learning process condition, and group 3 received both immobilization stress and underwent the learning process. For all three groups, sleep and awakening signals were recorded for each animal over a 2-hour period (baseline recording) using EEG and EMG recording. Immediately after the baseline recording was taken, the animals were subjected to immobilization stress for 2 hours. Immobilization stress was induced by placing the animals in a narrow (psychologic stress) with an uneven and stony floor (physical stress) for 2 hours. Then, poststress signals were recorded for each animal in group 1 for 2 hours. Meanwhile, the animals in the groups 2 and 3, were faced with a learning test (Barnes maze), before their post-stress signals were recorded. These experimental steps were repeated in each animal for 3 consecutive days. The baseline sleep and awakening signals were recorded every day, stating at 8:00 a.m. The average total time of sleep and awakening over 3 days were used to calculate percentage of total time each animal spent in REM and NREM sleep and awakening. The recorded values were considered as the baseline sleep patterns of each animal. As shown in Figure 2, we used EMG recording signals to identify the sleeping and awakening of the animals. That is low amplitude of EEG and high activity of EMG is associated to waking stage. Additionally, high amplitude of EEG and mild activity of EMG is associated to NREM stage. There are low amplitude of EEG and lowest level of EMG activity during REM sleep.

\section{Stress protocol}

We used the methods of Hoheisel et al. for stress with some modifications (16). Immobilization is a common stressor model used in the study of rodents. In the present study, the animals were placed in narrow Plexiglas boxes ( $16 \mathrm{~cm}$ long and $7 \mathrm{~cm}$ wide), which some stones placed on the floor. All rats were immobilized for 2 hours at the same time of day (during the light cycle) for 3 consecutive days.

\section{Electrophysiological recording of sleep}

Three permanent stainless-steel screws were implanted in the skull for EEG recording under ketamine $(65 \mathrm{mg} / \mathrm{kg})$ and xylazine $(15 \mathrm{mg} / \mathrm{kg})$ anesthesia. Additionally, Teflon coated stainless steel electrodes were placed subcutaneously on the neck muscles for EMG recording. Electrodes attached to a connector, which was cemented to rats head. After surgery, animals were allowed to recover for a week. For recording of EEG/EMG signals, an interconnect cable was used to connected the connector rats head to an EEG/EMG amplifier (eWave, Homemade, Science Beam Company, Tehran, Iran), and then the amplifier was connected to a PC with an AD converter. We used e-probe software for acquiring and analyzing the data (eProbe, version 7.2, Tehran, Iran) (17). Data were sampled at $3 \mathrm{kHz}$. Average REM, NREM sleep and waking condition EEG power was calculated for the (beta-like waves, $30 \mathrm{~Hz}, 100 \mu \mathrm{V}$ ), (slow-wave frequency or delta waves, $0.5-4 \mathrm{~Hz}, 150 \mu \mathrm{V}$ ) (beta waves, $30 \mathrm{~Hz},>100 \mu \mathrm{V})$, respectively. The slowwave power of each animal was normalized by expressing it as percentage of its average 2 hours' baseline. EEG and EMG were measured for 3 consecutive days during daylight, before and after stress exposure. At the first of experiment, baseline recording was performed for all groups on each day, then all animals were subjected to stress for 2 hours. After induction of stress, animals then immediately subjected to learning process (Barnes Test) and poststress recording. In the total length of our experimental period, all animals had full access to the food and water, except in the stress box and the Barnes test.

\section{The Barnes test assessing spatial learning and memory}

The Barnes maze test is a procedure for checking spatial memory $(\mathbf{1 7 , 1 8 )}$. The maze used in the current study consisted of a Plexiglas plate $(120 \mathrm{~cm}$ in diameter), with $3 \mathrm{~cm}$ between its edges and 18 holes 


\begin{tabular}{|c|c|c|c|c|}
\hline & Baseline recording & $\begin{array}{c}\text { Stress } \\
\text { (2 hours) }\end{array}$ & $\Longrightarrow$ Learning $\Longrightarrow$ & $\begin{array}{c}\text { Post-stress recordin } \\
\text { (2 hours) }\end{array}$ \\
\hline Group 1 & + & + & - & + \\
\hline Group 2 & + & - & + & + \\
\hline \multicolumn{5}{|l|}{ Group 3} \\
\hline Sham animals & + & - & + & + \\
\hline Stress animals & + & + & + & + \\
\hline
\end{tabular}

Figure 1. Experimental protocol in different three groups.
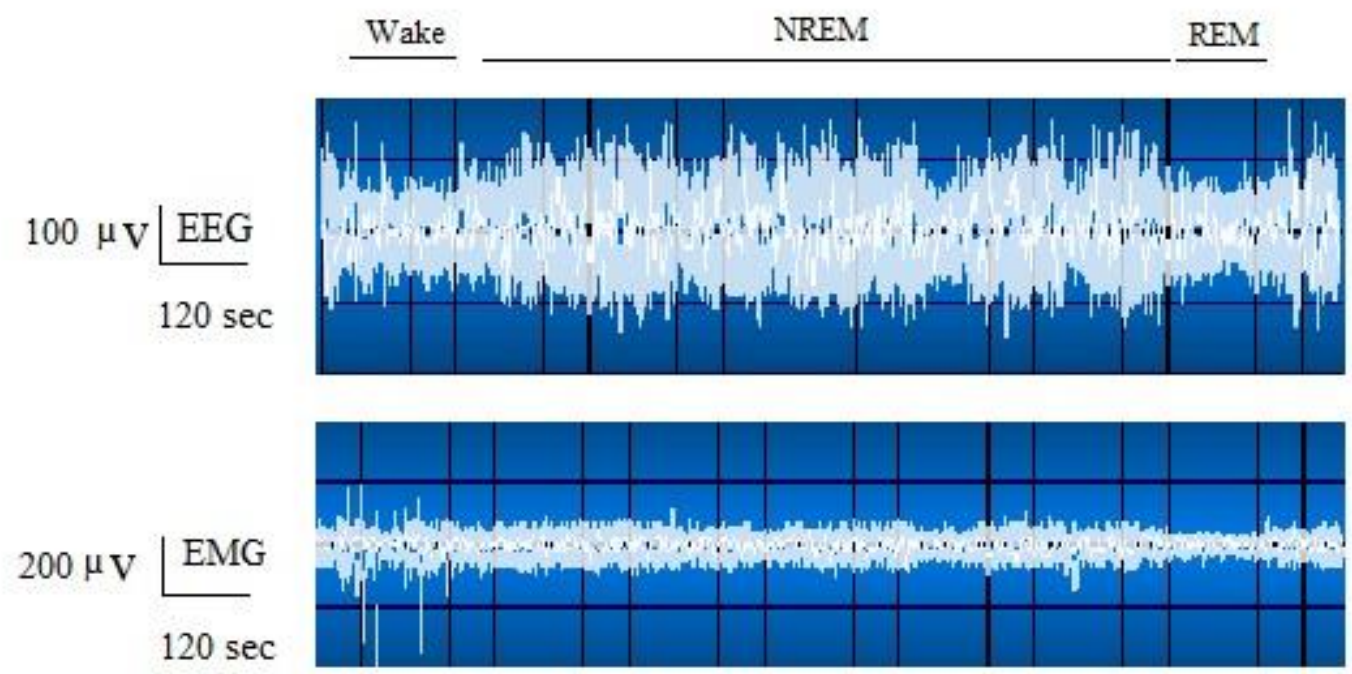

Figure 2. Demonstration of EEG and EMG variation during sleep-wake stages in rat. As shown in diagram, low amplitude of EEG and high activity of EMG illustrate waking stage. Furthermore, high amplitude of EEG and mild activity of EMG is associated with NREM stage. There are low amplitude of EEG and lowest level of EMG activity during REM sleep.

with a diameter of $10 \mathrm{~cm}$. A dark box (escape hole) made of black Plexiglas with dimensions of $20 \times 15$ $\times 10 \mathrm{~cm}$, was placed under one of holes. Four paper (A4 size) with different colored shapes were positioned on the laboratory wall to serve as visual cues. Two lamps $(150 \mathrm{~W})$, serving as annoying stimuli, were placed $110 \mathrm{~cm}$ above the top of the maze. Thirty minutes before the experiment, the animals were allowed to adapt to the environment. We put visual guide on the wall so that the animal could find the escape hole through them, with training. At the beginning of the experiment, the animals were placed in the center of the maze. Then, they freely moved in all directions until reaching the escape hole. The time to reach the escape hole (delay time) and the number of errors made when attempting to reach the hole were measured and evaluated. Three training sessions (each session consisted of 5 trials) were run on 3 consecutive days. The same procedure was repeated again on the fourth day (as a probe day) for the assessment of memory.

\section{Statistical analysis}

Statistical analyses were performed with the SPSS software (Statistical Package for the Social Sciences, version 20.0, SPSS Inc, Chicago, Illinois, USA). Data are expressed as mean \pm SEM. Additionally, paired $t$ test was used for statistical analysis (treatments against corresponding baseline (pre-stress) and post-stress against each other), and $\mathrm{p}<0.05$ was considered significant.

\section{Results}

Effects of stress on total time of REM, NREM and waking

The baseline recordings of the stress rats did not differ for any sleep parameter we examined. As shown in Figure (3A), immobilization stress resulted in significant post-stress decrease in REM sleep time that we examined $(\mathrm{p}<0.05)$. However, total time of NREM sleep significantly increased following stress as compared with baseline recording (pre-stress condition $)(p<0.05)$. Furthermore, immobilization 
stress could not have a significant effect on the time of awakening.

\section{Effects of learning on total time of REM, NREM and waking}

Undergoing the learning task resulted in a significant increase in post-learning REM sleep time when compared with the baseline (pre-learning) EEG recording $(\mathrm{p}<0.001$, Figure $3 \mathrm{~B})$. However, NREM sleep did not change markedly following the learning process. Interestingly, undergoing the learning process led to significant decreases in awakening time $(\mathrm{p}<0.05$, Figure $3 \mathrm{~B})$.

The effects of interaction of stress with learning on total time of REM, NREM and waking

Learning significantly increased post-stress total REM sleep time during three days ( 2 hours per day) compared to the baseline sleep (before stress exposure and learning processes) $(\mathrm{p}<0.05$, Figure 3C). However, learning process did not have any significant change in the post-stress total time of NREM sleep and awakening compared to the base line sleep (Figure 3C).
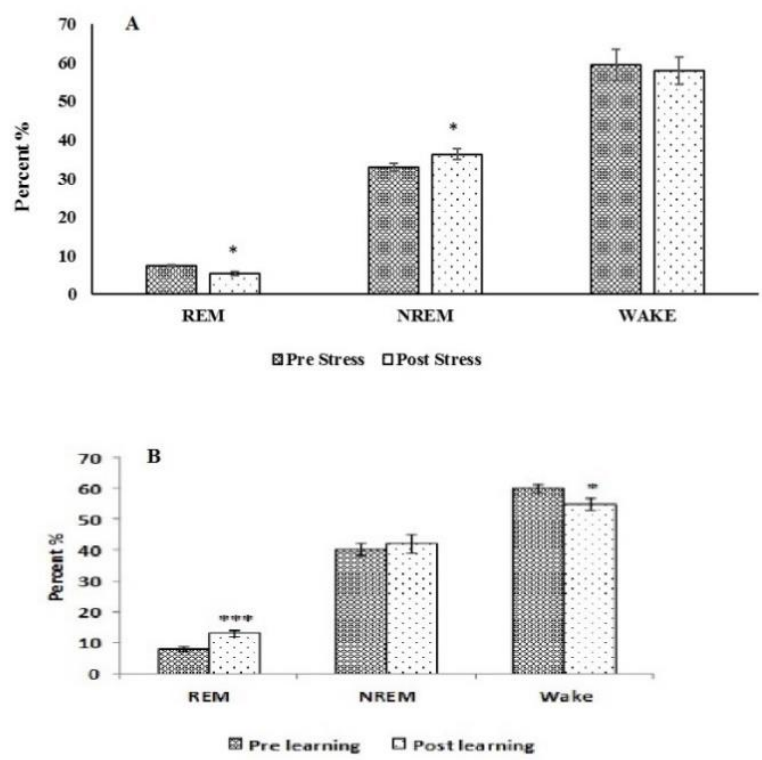

Comparison of sleep- wake alteration before and after the learning process or stress exposure

The difference between the baseline response and post-intervention response showed that stress caused a significant decrease in REM sleep (Figure 4). Additionally, learning, stress, and the interaction between learning and stress increased NREM sleep. However, there was no significant variation in these increments among the three groups. Similarly, no differences were found in the amount of wakefulness reduction between groups (Figure 4).

\section{The effects of stress on delay time and number} of errors in learning task

As shown in Figure 5, immobilization stress did not any significant change in the number of errors on days 1, 2 and 3 in learning task as compared with sham group (Figure 5A). However, the number of errors markedly increased in stress animals as compared with the sham group on probe day ( $<<0.05$, Figure 5A). Furthermore, immobilization stress significantly increased delay time on days 1 $(\mathrm{p}<0.05)$ and $2(\mathrm{p}<0.001)$ in learning task as compared with sham group. However, stress did not any significant change in the delay time on day 3 and probe day (Figure 5B). .

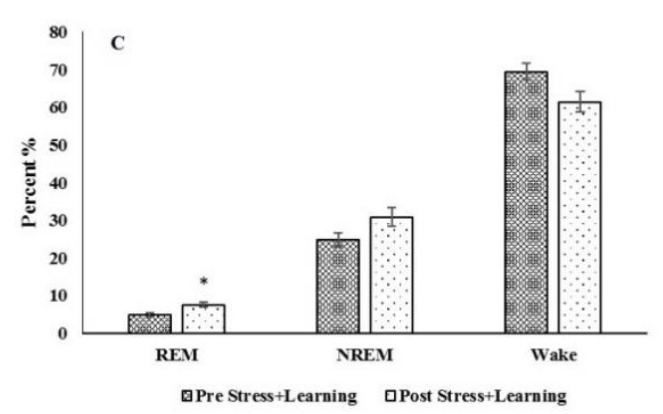

Figure 3. Effects of stress (A), learning paradigm (B), and interaction of stress with learning (C) on total time of REM, NREM and waking. Total time of signal recording was normalized as $100 \%$. Since, the sleep recordings were performed in the same animals, paired $t$ test was used for statistical analysis. ${ }^{*} \mathrm{p}<0.05$ and $* * * \mathrm{p}<0.001$ indicated significant differences with pre-stress, pre-learning, pre-stress + learning condition. 


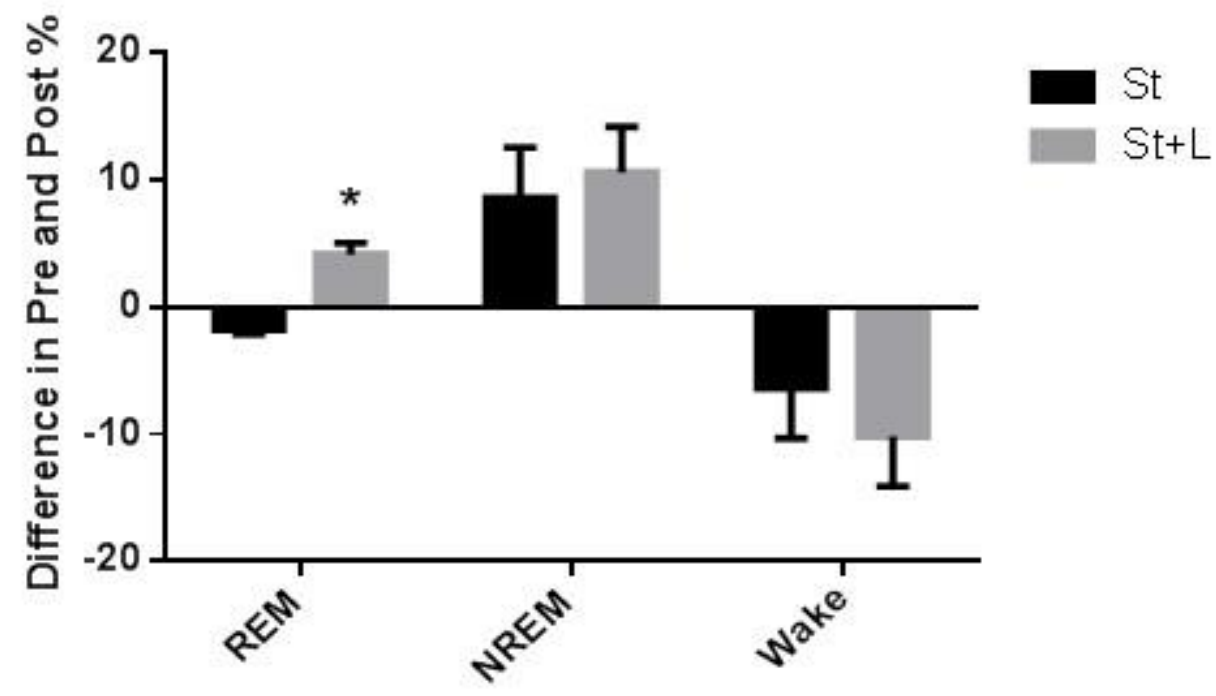

Figure 4. The difference value of REM, NREM and Wake stages before and after performance of learning or stress and both of these protocols. The graph indicates the significant decrease in REM sleep in stress exposed group $\left({ }^{*} \mathrm{p}<0.05\right)$. ${ }^{*} \mathrm{p}<0.05$ indicated significant differences with pre-stress + learning condition.
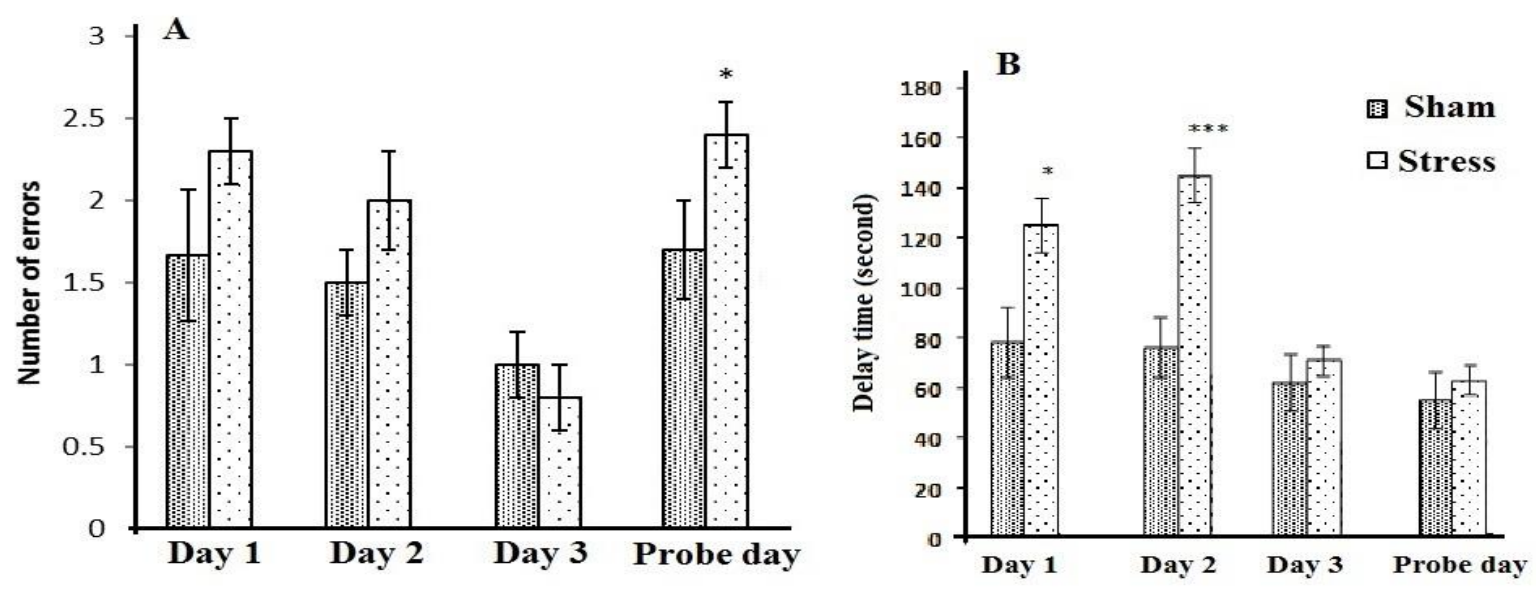

Figure 5. The effects of stress on (A) number of errors and (B) delay time and in learning task. Data are expressed as mean \pm SEM. Data analyzed using one-way analysis of variance (ANOVA) followed by post hoc LSD multiple comparison tests. Total time of signal recording was normalized as $100 \%$. ${ }^{\mathrm{p}}<0.05$ and $* * * \mathrm{p}<0.001$ indicated significant differences with sham group.

\section{Discussion}

In The first major finding of this study is that immobilization stress was followed by a significant decrease in total REM time, whereas our model of stress was followed by significant increases in total time of NREM. Furthermore, immobilization stress did not have a significant effect on the total time of awakening in our EEG recording. In our experiment, we used a model in which immobilization stress was induced in a narrow environment to induce psychological stress (19). Additionally, we used a modified model; specifically, the animals were placed in narrow plexiglass boxes with some stones placed on the floor to induce physical stress.

Ample evidence indicates that stress and sleep disturbances are often related (20). Sanford et al. (2010) identified that uncontrollable foot shocks shorten REM sleep, while controllable foot shocks increase REM sleep. Additionally, total NREM was decreased in mice only when shock training involved uncontrollable foot shocks (9). Moreover, cold stress (21) and social conflict (22) increase NREM sleep. Similarly, sleep after exposure to an open field or compartment for assessing intrinsic anxiety and search activity also indicate an initial decrease in REM sleep, followed by an increase (23). Therefore, stressor controllability is known to significantly affect sleep. It is doubtful that REM sleep involved basic activities that require low intelligence (24).

The second major finding of this study is that performance in the learning task (i.e., spatial learning via 
the Barnes maze test) was linked to a significant increase in total post-learning REM sleep. That is, directionally different markedly changes in REM occurred after the learning task than stress. However, total NREM sleep time did not change markedly following the learning process. So, the change in REM sleep is unlikely simply due to increased memory consolidation. REM sleep increases after learning, including after tow way active avoidance procedure (25-27), spatial learning in the Morris water maze (28), and operant learning (29).

In the present experiment, we attempted to determine how stress and learning influence total time spent in post-stress REM and NREM sleep, as well as total waking time, in rats. Therefore, our third major finding is that learning performance is related to an increase in total post-stress REM sleep time. However, learning did not significantly change the total post-stress NREM sleep and waking times. Thus, the increase in REM sleep after learning might be a functional response of the brain to the stress that is confronted during wakefulness.

Several studies have reported that post-training REM sleep is critical for efficient memory consolidation (30,31). Smith (2001) reported that REM sleep deprivation after a learning process results in memory deficits (32). Moreover, there is a high correlation between learning and REM sleep in the last sleep cycle before waking (30). Additionally, it has been shown that time spent in REM sleep increased following successful task acquisition (33). Smith and Lapp (1986) reported that the acquisition of a shuttle avoidance task induced a significant rise both in REM sleep time and the number of REM episodes, but there was no variation in REM density (34).

We further examine the effects of stress on delay time and the number of errors committed during the learning condition. Immobilization stress results in a significant increase in the number of errors only on probe day. Furthermore, immobilization stress significantly increased delay time in the learning task only on days 1 and 2 .

\section{Conclusion}

In conclusion, the data presented here suggest that immobilization stress leads to a decay in total REM sleep time. However, undergoing a learning process increased total post-stress REM sleep time. Thus, it seems that learning helps to overcome the inhibitory effects of stress on REM sleep.

\section{Acknowledgments}

Present study was supported by Neuroscience Research Center,
Baqiyatallah University of Medical Sciences.

\section{Conflict of Interest}

Authors declared no conflict of interests.

\section{References}

1. Day TA. Defining stress as a prelude to mapping its neurocircuitry: No help from allostasis. Prog NeuroPsychopharm Biol Psych. 2005; 29: 1195-2000. [DOI:10.1016/i.pnpbp.2005.08.005]

2. Ghobadi N, Sahraei H, Meftahi GH, Bananej M, Salehi S. Effect of estradiol replacement in ovariectomized NMRI mice in response to acute and chronic stress .J Apply Pharmaceutic Sci. 2016; 6(11): 176-84. [DOI:10.7324/JAPS.2016.601128]

3. Zarrin Ehteram B, Sahraei H, Meftahi GH, Khosravi M. Effect of intermittent feeding on gonadal function in male and female NMRI mice during chronic stress. Braz Arch Biol Technol. 2017; 60: e170607. [DOI:10.1590/1678-4324-2017160607]

4. Salehi Shemiran S, Meftahi GH, Sahraei H, Ghobadi N. Effect of testosterone replacement on feeding behaviors after acute and chronic stress in gonadectomized male NMRI mice. Front Biol. 2017; 12(6): 430-41. [DOI:10.1007/s11515-017-1470-2]

5. Korte SM, Koolhaas JM, Wingfield JC, McEwen BS. The Darwinian concept of stress: benefits of allostasis and costs of allostatic load and the trade-offs in health and disease. Neurosci Biobehav Rev. 2005; 29: 3-38. [DOI:10.1016/i.neubiorev.2004.08.009]

6. Machado DR, Afonso DJ, Kenny AR, et al. Identification of octopaminergic neurons that modulate sleep suppression by male sex drive. eLife. 2017; 6:e23130. [DOI:10.7554/eLife.23130]

7. Radahmadi M, Hosseini Dastgerdi A, Fallah N, Alaei H. The effects of acute, sub-chronic and chronic psychical stress on the brain electrical activity in male rats. Physiol Pharm 2017; 21(3): $185-92$.

8. Suchecki D, Tiba PA, Machado RB. REM sleep rebound as an adaptive response to stressful situations. Front Neurol. 2012; 3(41): 1-12. [DOI:10.3389/fneur.2012.00041]

9. Sanford LD, Yang L. Differential effects of controllable and uncontrollable footshock stress on sleep in mice. Sleep. 2010; 33:621-30. [DOI:10.1093/sleep/33.5.621]

10. Bonnet C, Leger L, Baubet V, Debilly G, Cespuglio R. Influence of a $1 \mathrm{~h}$ immobilization stress on sleep states and corticotrophin-like intermediate lobe peptide (CLIP or ACTH18-39, Ph-ACTH18-39) brain contents in the rat. Brain Res. 1997; 751: 54-63. [DOI:10.1016/S0006-8993(96)01390X]

11. Vazquez-Palacios G, Velazquez-Moctezuma J. Effect of electric foot shocks, immobilization, and corticosterone administration on the sleep-wake pattern in the rat. Physiol Behav. 2000; 71: 23-28. [DOI:10.1016/S0031-
[ 9384(00)00285-7]

12. Levin YI, Strygin KN, Korabelnikova EA. Effect of personality on changes of sleep structure caused by emotional stress. Human Physiology. 2002; 28(3): 37-42. [DOI:10.1023/A:1015548500861]

13. Pawlyk AC, Morrison AR, Ross RJ, Brennan FX. Stressinduced changes in sleep in rodents: models and mechanisms. 
Neurosci Biobehav Rev. 2008; 32(1): 99-117. [DOI:10.1016/i.neubiorev.2007.06.001]

14. Machida M, Yang L, Wellman LL, Sanford LD. Effects of stressor predictability on escape learning and sleep in mice. Sleep. 2013; 36(3): 421-30. [DOI:10.5665/sleep.2464]

15. Kim EJ, Dimsdale JE. The effect of psychosocial stress on sleep: A review of polysomnographic evidence. Behav Sleep $\begin{array}{lll}\text { Med. 2007; } & \text { 256-78 }\end{array}$ [DOI:10.1080/15402000701557383]

16. Hoheisel U, Vogt MA, Palme R, Gass P, Mense S. Immobilization stress sensitizes rat dorsal horn neurons having input from the low back. Eur J Pain. 2015;19(6): 861-70. [DOI:10.1002/ejp.682]

17. Erfani Sharifian F, Bahrami F, Zekri S, Sahraei H. Cinnamaldehyde antagonizes REM sleep reduction induced by immobilization stress in rats. Mazandaran Univ Med Sci . 2019; 29 (175): 14-24.

18. Hadipour MM, Kaka GR, Bahrami F, et al. Crocin improved amyloid beta induced long-term potentiation and memory deficits in the hippocampal CA1 neurons in freely moving rats. Synapse. 2018; 72(5): e22026. [DOI:10.1002/syn.22026]

19. Buynitsky T, Mostofsky DI. Restraint stress in biobehavioral research: Recent developments. Neurosci Biobehav Rev. 2009; $\quad 33(7)$ : 1089-98 [DOI:10.1016/j.neubiorev.2009.05.004]

20. Vargas I, Friedman NP, Drake CL. Vulnerability to stressrelated sleep disturbance and insomnia: Investigating the link with comorbid depressive symptoms. Trans Issues Psychol Sci. 2015; 1(1): 57-66 [DOI:10.1037/tps0000015]

21. Palma BD, Suchecki D, Tufik S. Differential effects of acute cold and footshock on the sleep of rats. Brain Res. 2000; 861(1): 97-104. [DOI:10.1016/S0006-8993(00)02024-2]

22. Meerlo P, Pragt BJ, Daan S. Social stress induces high intensity sleep in rats. Neurosci Lett. 1997; 225(1): 41-44. [DOI:10.1016/S0304-3940(97)00180-8]

23. Tang X, Xiao J, Liu X, Sanford L D. Strain differences in the influence of open field exposure on sleep in mice. Behav. Brain Res. 2004; 154: 137-47. [DOI:10.1016/j.bbr.2004.02.002]

24. Smith CT, Nixon MR, Nader RS. Posttraining increases in REM sleep intensity implicate REM sleep in memory processing and provide a biological marker of learning potential. Learn Mem. 2004; 11(6): 714-19. [DOI:10.1101/lm.74904]

25. Smith C, Young J, Young W. Prolonged increases in paradoxical sleep during and after avoidance-task acquisition. Sleep. 1980; 3:67-81.

26. Portell-Cortes I, Marti-Nicolovius M, Segura-Torres P, Morgado-Bernal I. Correlations between paradoxical sleep and shuttle-box conditioning in rats. Behav Neurosci. 1989; 103: 984-90. [DOI:10.1037/0735-7044.103.5.984]

27. Datta S, Saha S, Prutzman S L, Mullins O J, Mavanji V. Pontine-wave generator activation-dependent memory processing of avoidance learning involves the dorsal hippocampus in the rat. J Neurosci Res. 2005; 80: 727-737. [DOI:10.1002/inr.20501]

28. Smith C, Rose G M. Posttraining paradoxical sleep in rats is increased after spatial learning in the Morris water maze. Behav Neurosci. 1997; 111: 1197-204. [DOI:10.1037/07357044.111.6.1197]

29. Smith C, Wong P T. Paradoxical sleep increases predict successful learning in a complex operant task. Behav Neurosci. 1991; 105: 282-88. [DOI:10.1037/0735-7044.105.2.282]

30. Stickgold R, Whidbee D, Schirmer B, Patel V, Hobson JA. Visual discrimination task improvement: A multistep process occurring during sleep. J Cog Neurosci. 2000; 12: 246-54. [DOI:10.1162/089892900562075]

31. Louie K, Wilson MA. Temporally structured replay of awake hippocampal ensemble activity during rapid eye movement sleep. Neuron. 2001; 29: 145-56. [DOI:10.1016/S08966273(01)00186-6]

32. Smith CT. Sleep states and memory processes in humans: Procedural vs. declarative memory systems. Sleep Med Rev. 2001; 5: 491-506. [DOI:10.1053/smrv.2001.0164]

33. Smith CT. Sleep states, memory processes and synaptic plasticity. Behav Brain Res. 1996; 78: 49-56. [DOI:10.1016/0166-4328(95)00218-9]

34. Smith C, Lapp L. Prolonged increases in both PS and number of REMs following a shuttle avoidance task. Physiol Behav. 1986; 36: 1053-57. [DOI:10.1016/0031-9384(86)90479-8]

\section{How to Cite This Article:}

Erfani Sharifian F, Bahrami F, Bahari Z. Spatial Learning Paradigm Can Increase Post-Stress Total Time of REM Sleep in Immobilized Rats. J Adv Med Biomed Res. 2019; 27 (123) :9-15

Download citation:

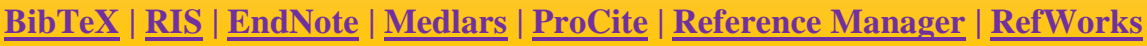

Send citation to:

9. Mendeley $2 \underline{\text { Zotero }}$ (i) RefWorks $\underline{\text { RefWorks }}$ 